\title{
SURFACE DEGRADATION OF NANOCRYSTALLINE ZIRCONIA DENTAL IMPLANTS
}

\author{
VÁCLAV OCELÍK ${ }^{1}$, ULF SCHEPKE ${ }^{2}$, HAMID HAJI RASOUL ${ }^{2}$, \\ MARCO S. CUNE ${ }^{2} \&$ JEFF TH.M. DE HOSSON ${ }^{1}$ \\ ${ }^{1}$ Department of Applied Physics, Zernike Institute for Advanced Materials, University of Groningen, \\ The Netherlands \\ ${ }^{2}$ Department of Fixed and Removable Prosthodontics and Biomaterials, University of Groningen, \\ The Netherlands
}

\begin{abstract}
Yttria-stabilized zirconia prepared by hot isostatic pressing represents attractive material for biomedical applications. In this work the degradation of yttria-stabilized zirconia dental implants abutments due to the tetragonal to monoclinic phase transformation after one year of clinical use was studied in detail. Microstructural characterization by Electron Back Scattering Diffraction was successfully applied. The amount and distribution of the monoclinic phase, the grain-size distribution and crystallographic orientations between tetragonal and monoclinic crystals in $3 \mathrm{~mol} \%$ yttria-stabilized polycrystalline zirconia were determined in two different types of abutments currently used in clinical practice. Clear crystallographic orientation relationship between parent tetragonal and daughter monoclinic phase was clearly observed. An important and novel conclusion is that no substantial bulk degradation of 3Y-TZP dental implant abutments was detected after 1 year of clinical use.
\end{abstract}

Keywords: dental implants, yttria-stabilized zirconia, degradation, nano-crystalline, electron backscatter diffraction.

\section{INTRODUCTION}

Zirconia $\left(\mathrm{ZrO}_{2}\right)$ belongs to an important class of industrial ceramic materials for structural applications due to its mechanical performance [1]. The most structural engineering and biomedical applications utilize its tetragonal and cubic phases. The fact these phases are not stable at low temperatures requires zirconia doping with oxides such as $\mathrm{Y}_{2} \mathrm{O}_{3}$ which stabilize these high-temperature phases at room temperature or at temperature in the human body. The stabilization effect is due to the oxygen vacancy concentration which originates from the introduction of $\mathrm{Y}_{2} \mathrm{O}_{3}$ to $\mathrm{ZrO}_{2}$ [2]. Another way of stabilizing the tetragonal structure at room temperature is the formation of nano-crystalline specimens in a sintered form [1]. The tetragonal phase is preferred when a high toughness is required. Within the field of dentistry $\mathrm{Y}_{2} \mathrm{O}_{3}$ stabilized $\mathrm{ZrO}_{2}$ is used as a base material for indirect restorations (i.e. crowns and bridges), for dental implants and implant abutments [3]. $\mathrm{Y}_{2} \mathrm{O}_{3}$ stabilized $\mathrm{ZrO}_{2}$ has a white appearance and can be colored easily which is, in contrast to metals, of an aesthetic advantage [4]. Also, its use allows CAD-CAM production of dental restorations [5]. However, the lifetime of widely used 3 mol.\% yttria-stabilized tetragonal zirconia poly-crystals (3Y-TZP) for these biomedical applications is rather unknown and, is predominantly estimated on the basis of in vitro accelerated ageing experiments [6]. Here, the intraoral conditions during clinical use are poorly mimicked, resulting in a doubtful and questionable external validity. In fact, it is a major source of concern.

The structurally detrimental phase transformation due to spontaneous increase of the amount of the brittle monoclinic phase is known as low-temperature degradation (LTD) [7], because this aging process of the material occurs at moderate temperatures below $400^{\circ} \mathrm{C}$. The influence of water or water vapor on this transformation was also studied, [8]. However, the kinetics, rate constants and other details such as the possible difference between the attack by water vapor and liquid water remain unknown. 
This study is aimed at determining in detail whether the currently used polycrystalline yttria-stabilized tetragonal zirconia (3Y-TZP) dental implant abutments are susceptible to LTD at temperature of human body in a clinical setting (i.e. screw retained to a titanium implant on the bone-side and bonded to a full crown on the oral side). A small surface area between the connecting parts was constantly in contact with the oral environment. The location of the EBSD analysis was chosen according to the assumed contact stress peaks inside the material (Fig. 1).

In particular Electron Backscatter Diffraction is explored in the structural analysis of these phenomena since its resolution is of the order of 40-50 nanometers [9]. In addition, this method provides access to crystal orientation correlations, representation of texture and grain boundary character distributions via grain orientation maps. Samples of 3Y-TZP dental abutments were removed, after one year of clinical use, and examined on t-m transformation. Two types of zirconia sintered dental abutments were tested: Atlantis ${ }^{\mathrm{TM}}$ CAD-CAM designed and ZirDesign ${ }^{\mathrm{TM}}$ stock specimen, both from one and the same producer (DentsplySirona Implants, Mölndal, Sweden). Pristine, geometrically identical samples were conserved in a dry environment at room temperature for pairwise comparison. The amount of $\mathrm{m}-\mathrm{ZrO}_{2}$ phase was determined using electron backscatter diffraction (EBSD). Experimental methods to study phase transformations in $\mathrm{ZrO}_{2}$ with a focus mainly on hip prostheses are summarized in [7]. Traditionally this type of study has been carried out using neutron diffraction and/or electron diffraction in a transmission electron microscopy (TEM). The most extensively used technique in literature is X-ray diffraction (XRD) [10]. The latter method is a fast and a nondestructive method, allowing the monitoring of LTD evolution on the same specimen. Nonetheless, XRD is limited as far as the lateral resolution is concerned and the typical spot size is of the order of millimeters. X-ray powder diffraction is best for identification of homogeneous and single-phase materials. It is also not very precise for a monoclinic phase content lower than 5\%, making it not suitable for monitoring the onset of the transformation [7].

\section{EXPERIMENTAL METHODS}

Four samples were retrieved from 4 patients who were treated for premolar replacement at the Center for Dentistry and Oral Hygiene of the University Medical Center Groningen; the Netherlands. These patients received a single crown on a dental implant. The dental abutments were used to connect the dental implants and the crowns. 2 types of dental abutments were used, standard stock abutments (ZirDesign) and individually computer aided designed and manufactured abutments (Atlantis). After one year of clinical use the dental abutments were replaced. The control group consists of 4 pristine dental abutments of the same types, with the same anatomy, preserved in a dry environment at room temperature during the same time as for clinical samples.

Sample preparation procedure for microstructural observations consists of filling the sample holes using conductive Demotec70 epoxy. After drying, the samples were fixed in conductive $\mathrm{Cu}$ based mold. Mechanical grinding with $\mathrm{SiC} 180$ grade powder under water was used until the required cross section was exposed. Further grinding using Struers Piano 220 and 1200 magnetic disks was performed before lapping by 9, 6, 3 and $1 \mu \mathrm{m}$ diamond particle suspensions (each about 5 minutes). Final polishing by Struers OPS silica suspension $(40 \mathrm{~nm})$ for 10 minutes was used to prepare surface of zirconia implants for EBSD observation.

EBSD microstructural characterization was conducted using a Philips XL30 FEG scanning electron microscope operated at standard high vacuum mode $\left(5.510^{-5} \mathrm{mBar}\right)$ 


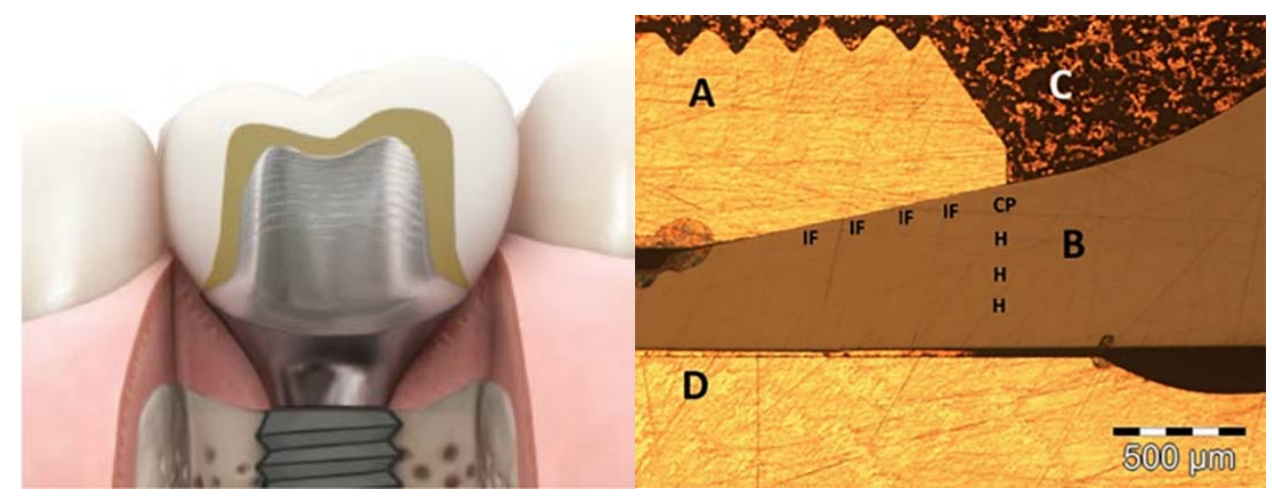

Figure 1: Left: the role of abutment in Atlantis patient-specific dental implant (according [11]). Right: light microscopy image from one part of 3Y-TZP embedded abutment (area B) after grinding and before its final polishing. Area (A) marks external thread of the dental implant from Ti alloy. Area (C) marks copper based conductive mount and area (D) marks inner screw of dental implant. Approximate locations of EBSD scans are marked as CP, IFs and Hs, respectively (see text for details). Another 8 scans were collected on other rotation symmetrical side of abutment to get to the total of 16 scans for each sample. The CP scans were made in area of $25 \times 25 \mu \mathrm{m}$. The $\mathrm{H}$ and IF scans were made in area of $5 \times 5 \mu \mathrm{m}^{2}$.

equipped with TSL EDAX Electron Back Scatter Diffraction system containing DigiView 3 CCD camera.

In total sixteen scans were collected from each abutment sample. The specimens were embedded in such a way that the buccal and the lingual side of the implant abutment could be scanned. Because of the time-consuming scanning procedure, the following areas, shown in Fig. 1 were selected for EBSD scans: I. Contact point (CP) between the dental abutment and the dental implant, as this area is assumable the area of the highest stress concentration built-up during the service; II. Four areas at the interface along the dental implant (IF) in the apical direction, and III. Three areas $(\mathrm{H})$ from contact point towards the axis of the dental implant. The $\mathrm{CP}$ were scans with size of $25 \times 25 \mu \mathrm{m}^{2}$ as close as possible to the dental implant $(\sim 200 \mathrm{~nm})$ with the distance between scanning points of $50 \mathrm{~nm}$. Four IF scans were made, also as close as possible to the dental implant $(\sim 200 \mathrm{~nm})$, with an equal distance between the scan areas of $100 \mu \mathrm{m}$. Three $\mathrm{H}$ scans were made with an equal distance of $100 \mu \mathrm{m}$ between them and as close as possible to the CP scan. The IF and H scans are $5 \times 5 \mathrm{~m}^{2}$ in size with distance between scanning points of $40 \mathrm{~nm}$. Hexagonal grid of point's arrangement was selected for all scans. Data are presented in different types of orientation imaging microscopy (OIM) maps, showing grain orientations, type of phase and grain boundaries.

EDAX TSL OIM Data Collection software v7.3 was used for collecting the OIM data. The electron beam acceleration voltage was set to $10 \mathrm{kV}$ with the beam current of $2 \mathrm{nA}$. This beam setting suppresses a tendency of the non-conductive ceramic sample to charge and distort collected EBSD maps and also allows achieving higher lateral resolution required for samples with small grains $(\sim 500 \mathrm{~nm})$. CCD camera pattern binning of $8 \times 8$ allows the EBSD patterns collection and indexing speed of 70 frames per second resulting in a final time for one EBSD scan of 70 minutes for large scan of $25 \times 25 \mu \mathrm{m}^{2}$ area and about 4-5 min for scan with size of $5 \times 5 \mu \mathrm{m}^{2}$, respectively. 


\section{RESULTS AND DISCUSSION}

Fig. 2 shows typical [001] Inverse pole figure (IPF) maps and Phase maps collected on the Atlantis CAD-CAM and ZirDesign samples that had functioned clinically for one year. Maps show the same CP sample area with size of $25 \times 25 \mu \mathrm{m}^{2}$, containing about 290k points with distance of $50 \mathrm{~nm}$ between them. More than $99.8 \%$ points were successfully indexed during collection of EBSD map, but only points with probability of correct indexing higher than $85 \%$ are colored. All maps in Fig. 2 are combined with Image Quality (IQ) signal (pattern contrast map) represented by points brightness. From insets located at top right corners one may conclude that the IQ parameter is higher at the centers of $\mathrm{t}-\mathrm{ZrO}_{2}$ grains and decreases slightly at tetragonal phase grain boundaries but substantially inside monoclinic phase. Tetragonal grains have sub-micrometer size and monoclinic phase grains are even smaller. As Fig. 2 clearly demonstrates, the one year clinically tested Atlantis sample contains less than $1 \%$ of monoclinic phase whilst ZirDesign sample also after one year of clinical use shows more than $6 \%$ of monoclinic $\mathrm{ZrO}_{2}$ phase. Detailed insets in phase maps in Fig. 2 clearly demonstrate that $\mathrm{t}-\mathrm{ZrO}_{2}$ phase nucleates at grain boundaries and in triple points of tetragonal grains.

Table 1 summarizes the average amounts of tetragonal phase detected in both type of samples and also make a comparison between the used and not used abutments. Averaging has been done over all 16 locations on two samples tested in each group. 95\% confidence intervals of the mean are also indicated in Table 1. It has to be noted that the main source of variance has been observed between two samples and not between different places of EBSD scans indicated in Fig. 1.

Statistical testing rejected the hypothesis about the influence of 1 year clinical use of abutment on the amount of monoclinic phase for both type of materials. A negative result was also obtained when evaluating a hypothesis of the influence of a different place on the sample as regards the amount of observed monoclinic phase. Therefore, a general conclusion could be made that the amount of monoclinic phase does not increase in both type of tested abutments during one year of clinical use.

Nevertheless, a positive conclusion is that careful EBSD mapping could be used to measure a rather small amount of monoclinic phase in nano-crystalline 3Y-TZP dental abutments. An attempt to confirm this EBSD observed $\mathrm{m}-\mathrm{ZrO}_{2}$ phase amounts in the same samples by standard XRD diffraction measurement according [10], failed due to lack of sensitivity of detecting of monoclinic phase peaks in the diffraction spectrum.

However, from the results shown in Table 1 one may conclude that in general ZirDesign abutments contain more $\mathrm{m}-\mathrm{ZrO} 2$ phase than Atlantis ones. However, all measured amounts of monoclinic phase in our experiment were far below the value when a degradation of mechanical properties is expected. The amount of monoclinic phase measured during accelerated hot steam test [10], starts to exceed $10 \mathrm{vol} \%$ after a few hours at an increased temperature and authors suggested to use optical interferometry and atomic force microscopy

Table 1: Comparison of the mean amount (in vol\%) of monoclinic $\mathrm{ZrO}_{2}$ phase in used and not used samples for both type of dental abutments. 95\% of confidence intervals for mean values are also indicated.

\begin{tabular}{lcc}
\hline Sample & Not used & Used \\
\hline Atlantis & $0.57 \pm 0.27$ & $1.0 \pm 0.27$ \\
ZirDesign & $6.17 \pm 1.21$ & $3.8 \pm 1.30$ \\
\hline
\end{tabular}




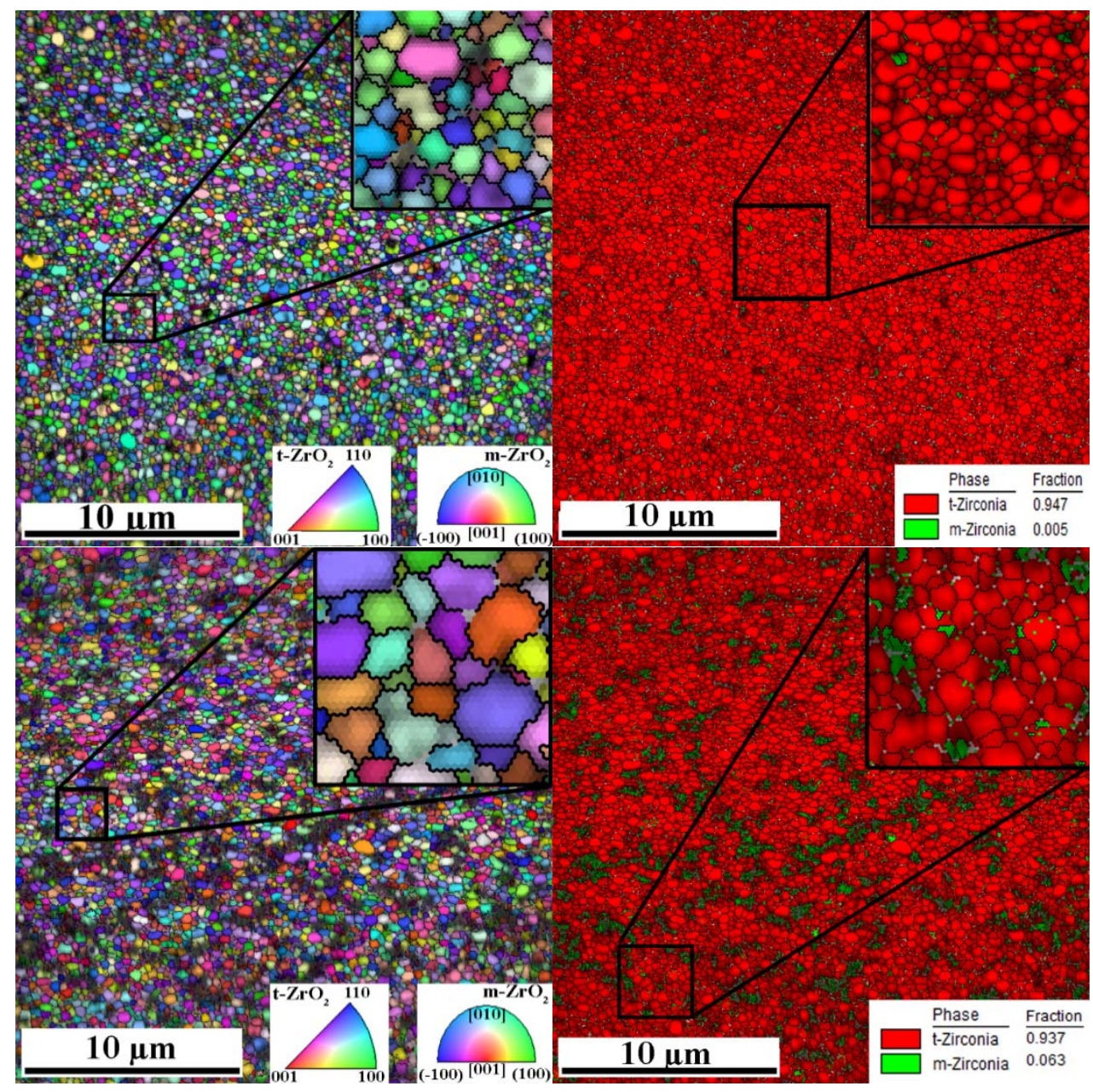

Figure 2: [001] IPF map (left) and phase map (right) combined with IQ map of one year used Atlantis (top) and ZirDesign (bottom) samples at CP locations. Grain boundaries $\left(>5^{\circ}\right)$ are shown as black lines.

accelerated hot steam test [10], starts to exceed $10 \mathrm{vol} \%$ after a few hours at an increased temperature and authors suggested to use optical interferometry and atomic force microscopy for the detection of initial stage of transformation, when small amounts of monoclinic phase should be detected.

Our results confirmed that EBSD is another appropriate method with an advantage of additional crystallographic information. Due to very careful sample preparation consisting of gradual grinding and polishing we do not expect that the detected monoclinic phase could develop during this procedure.

Fig. 3 compares grain size distributions calculated from OIM maps for both phases in both types of samples. ZirDesign samples show a larger average grain size of tetragonal phase $(410 \mathrm{~nm})$ in comparison with Atlantis abutments $(340 \mathrm{~nm})$. Grain size distributions of tetragonal grains for both type samples are very close to a log-normal distribution with a little 

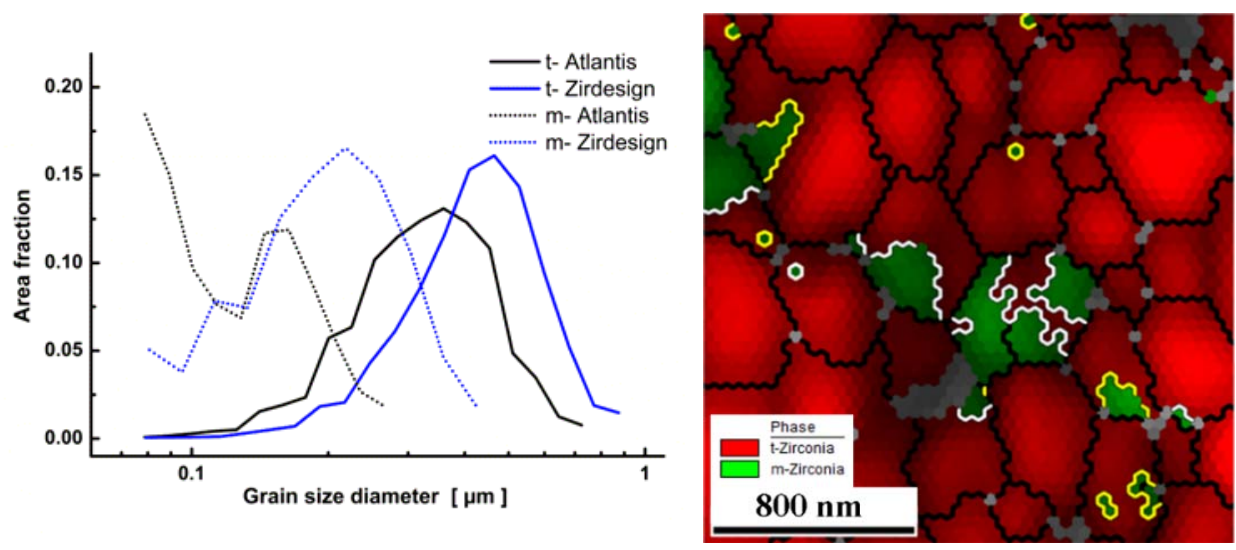

Figure 3: Left: Grain size distributions calculated for tetragonal and monoclinic grains (misorientation $>5^{\circ}$ ) of both type of specimens. Right: Detail of Phase map combined with IQ map and grain boundaries observed on ZirDesign sample. Grain boundaries with misorientation larger than $5^{\circ}$ are marked by black lines. Grain boundaries with special misorientation angles are highlighted by color lines: $97.5^{\circ} \pm 1^{\circ}$ in white and $44.6^{\circ} \pm 1^{\circ}$ in yellow color, respectively.

bit larger dispersion in Atlantis material. The average size of monoclinic phase grains is only a fraction from the size of the tetragonal grains, which confirms the fact that monoclinic phase nucleates at grain boundaries and the triple junctions between tetragonal grains and propagates into them, as seen in the phase map insets in Fig. 2. Smaller amounts of the monoclinic phase in Atlantis material could be related to its smaller grain size. It is known that a smaller grain size enhances the stability of the tetragonal phase [7]. The experimentally observed lack of increase of the amount of monoclinic phase in the specimens was indirectly confirmed by the fact, that also no substantial change of grain size distribution between used and not-used sample was found. An observable shift towards a smaller grain distribution was observed by EBSD on artificially aged $\mathrm{ZrO}_{2}$ samples in autoclave [12], which is obvious when only a small part of tetragonal grain transforms to monoclinic form.

Crystal orientation data collected during EBSD scanning allows a more detailed study of tetragonal to monoclinic phase transformation. A special distribution of misorientation angles between neighboring tetragonal and monoclinic grains was detected on all samples. Two special misorientation angles with a high presence rate were detected with maxima at 44.6 and 97.5 degrees, respectively. The right side of Fig. 3 shows a detailed part of the microstructure of ZirDesign sample. The phase map is combined with IQ map and grain boundaries are highlighted by colored lines.

General grain boundaries $\left(>5^{\circ}\right)$ are black, whereas grain boundaries with special angles mentioned above are marked by yellow and white color, respectively. Fig. 3 demonstrates clearly as it was observed on all four types of samples: almost every monoclinic $\mathrm{ZrO}_{2}$ grain has just one tetragonal $\mathrm{ZrO}_{2}$ neighboring grain with the misorientation angle of $44.6 \pm 1$ or $97.5 \pm 1$ degrees. Assuming that a transformation from tetragonal to monoclinic phase occurred, a relation between parent and daughter grain becomes evident. Monoclinic grain grew mainly from triple junctions of $\mathrm{t}-\mathrm{ZrO}_{2}$ grain boundaries and proceeded into the parent $\mathrm{t}-\mathrm{ZrO}_{2}$ grain. 


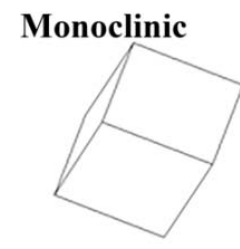

Tetragonal

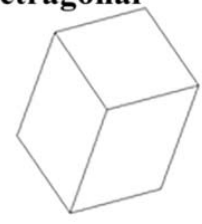

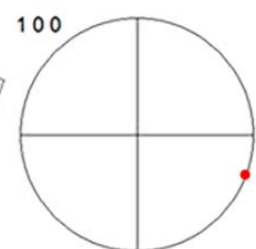

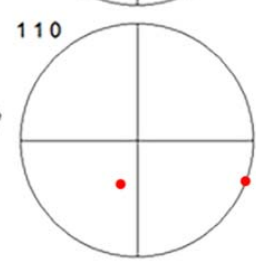

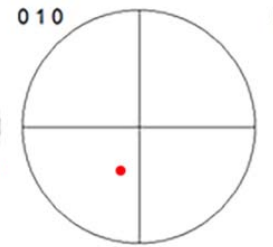
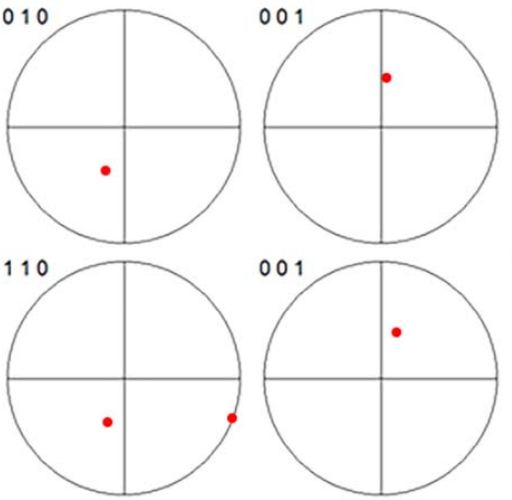

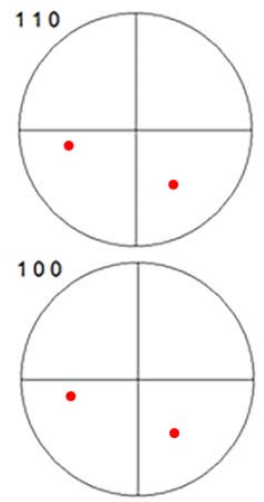

Figure 4: Pole figure plots of different planes for daughter (monoclinic) and parent (tetragonal) grains having misorientation angle of $46.4^{\circ}$ for demonstration OR in eqn (1).

The sharp misorientation angles detected between parent $\mathrm{t}-\mathrm{ZrO}_{2}$ and daughter $\mathrm{m}-\mathrm{ZrO} \mathrm{r}_{2}$ phase indicate the existence of crystallographic orientation relationship(s) (ORs) associated with t-m transformation of 3Y-TZP phase. The character of these relationship have been revealed by observing a substantial amount of mutual pole figure plots of parent tetragonal and daughter monoclinic grain having one of these two misorientation angles. A pole figure is a graphical representation of the orientation of crystal in space. Stereographic projection is used to plot poles - the intersections of selected crystal planes normal with a sphere. Fig. 4 compares pole figure plots for parent and daughter grain having misorientation angle close to 44.6 degrees. On more than 15 such pairs the same OR has been observed for all pairs having this misorientation angle:

$$
[100]_{\mathrm{m}} \|[110]_{\mathrm{t}} \text { and }[010]_{\mathrm{m}} \|[110]_{\mathrm{t}} \text { and }[001]_{\mathrm{m}} \|_{\mathrm{c}}[001]_{\mathrm{t}} \text { and }(100)_{\mathrm{m}} \|(100)_{\mathrm{t}},
$$

where $\|$ means that directions or planes are parallel and $\|_{c}$ denotes they are almost parallel. The average of small angle between [001] directions in the coupled tetragonal and monoclinic crystals were measured as $8.8^{\circ}$ with standard deviation of $0.6^{\circ}$. This value is in agreement with the inclination of unit cell angle $\beta$ from the perpendicular direction, being $99.215^{\circ}$ for monoclinic $\mathrm{ZrO}_{2}$ phase [13].

However, for monoclinic and tetragonal parent-daughter crystal pairs with misorientation angle of $97.5^{\circ}$ situation is more complicated and one of the following two different variants of ORs was detected:

$$
\begin{aligned}
& (100)_{\mathrm{m}} \|(110)_{\mathrm{t}} \text { and }[010]_{\mathrm{m}} \|[001]_{\mathrm{t}} \text { and }(001)_{\mathrm{m}} \|_{\mathrm{c}}(110)_{\mathrm{t}} \text { and }(101)_{\mathrm{m}} \|_{\mathrm{c}}(010)_{\mathrm{t}} \\
& (100)_{\mathrm{m}} \|(001)_{\mathrm{t}} \text { and }[010]_{\mathrm{m}} \|[110]_{\mathrm{t}} \text { and }(001)_{\mathrm{m}} \|_{\mathrm{c}}(110)_{\mathrm{t}} \text { and }(011)_{\mathrm{m}} \|_{\mathrm{c}}(100)_{\mathrm{t}}
\end{aligned}
$$

Simultaneous EBSD mapping observations of different crystal modifications inside an $\mathrm{MgO}$ stabilized zirconia particles embedded in steel matrix were already observed [14]. Unfortunately, no information about crystallographic orientation relationships between them has been provided. On the base of theoretically predicted possible orientation relationships between parent tetragonal and daughter monoclinic $\mathrm{ZrO}_{2}$ phase [15], Cayron et al. [16], were able to reconstruct orientations of primary tetragonal grains inside the fully transformed monoclinic microstructure. Orientation relationships between parent tetragonal and daughter 
monoclinic phase suggest that the transformation has a martensitic character although not only ORs predicted from simple lattice distortions [15], were detected.

Finally the texture (preferred crystal orientation in the sample) detected in both type of dental abutments was examined in detail. The textures observed are shown in Fig. 5 for both types of abutments in the form of texture plots. A texture plot is a type of pole figure plot in which all detected crystal directions are shown in a single figure, i.e. in the form of local density of poles. Random texture should result in a uniform density of poles. On the contrary, texture plots of tetragonal $\mathrm{ZrO}_{2}$ grains shown in Fig. 5 are both characterized by a preferential orientation of the (001) plane normal parallel to the samples surface normal A3, which is in accordance to the tangential direction towards rotation axes symmetry of the dental abutment. This texture is slightly stronger in the ZirDesign sample, expressed by higher value (3.98) of multiples of uniform density 1 in (001) texture plot. This result is in contradiction with the recently published EBSD observations [17], on commercial hipped dental 3Y-TZP ceramics, where no texture was reported. However, in comparison with our results (Fig. 2) a relatively low quality OIM map was presented in [17], and no information is provided concerning the characterization of the tetragonal crystal phase used for indexing of the Kikuchi patterns. Because the authors did not mention any complexity with pseudo-symmetry during automatic indexing, we suspect that the cubic instead of tetragonal phase was used for indexing and therefore the preferential orientation of an elongated tetragonal axis $c$ could not be detected. In comparison with our results also a larger average grain size has been observed on level of $500 \mathrm{~nm}$.

In this study, we have clearly demonstrated that EBSD proves an experimental tool to bridge the gap between the very local information provided with transmission electron microscopy and the volume averaging information that results from X-ray diffraction. It is shown that EBSD is an efficient way to study zirconia-based materials for biomedical applications, their stability and phase transformations. A possibility to realize EBSD observations using low-vacuum scanning electron microscopes [18], and the rapid scanning offered by the fast acquisition cameras [19], allow EBSD mapping of zirconia without any deleterious charging effects.

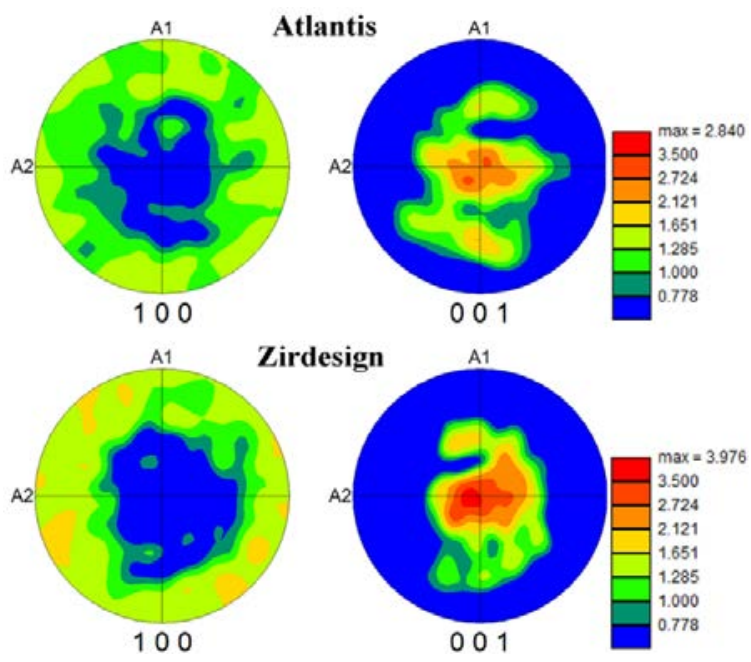

Figure 5: (100) and (001) Pole figure texture plots calculated from EBSD scans performed on Atlantis and ZirDesign 3Y-TZP dental abutments. 
There are only a few studies in literature where the EBSD approach was used to study the microstructure of 3Y-TZP[12,20-23]. Often the drawbacks due to charging effects are circumvented by adding a thin carbon coating or by lowering the SEM voltage during OIM scan. In this study we have found that on a well (mechanically) polished surface of 3Y-TZP the combination of SEM voltage reduction $(10 \mathrm{kV})$ and fast OIM scanning ( $>60 \mathrm{fps})$ could result in mapping areas large enough for nanostructured materials (more than 5000 grains). High quality of Kikuchi pattern allows reliably distinguish tetragonal and monoclinic $\mathrm{ZrO}_{2}$ phase and also to study orientation relationship between them. However, complications due to pseudo-symmetry during automatic pattern indexing (similar as in [22], [24]) requires $90 @<110>$ harmonization of grain orientation to obtain correct grain size distributions.

The fact that we did not observe an increasing concentration of monoclinic phase after one year of clinical test could be understood within the framework of long-time aging studies of tetragonal 3Y-TZP at temperature of human body [25], [26]. The phenomenon of tetragonal to monoclinic transformation as detected by X-ray micro-diffraction and confirmed by SEM observations is considered being due to the surface exposed to humidity. After one year the transformed layer attains a thickness less than $1 \mu \mathrm{m}$. However, our sample preparation procedure does not allow examining areas very close to the outer surfaces exposed to the environmental conditions. Only 1-2 grains at the edge of our scans were directly exposed to the humid conditions and these are statistically less relevant in our observation. Our measurements are therefore more relevant for a characterization of bulk microstructure of dental abutments mainly after their production. However, importantly our measurements confirm that bulk characteristics of 3Y-TZP are not changed during one year of clinical function.

\section{CONCLUSIONS}

Electron backscatter diffraction in a scanning electron microscope has been used to study the stability of yttria-stabilized $\mathrm{ZrO}_{2}$ dental implants abutments that had functioned clinically for one year. The overall conclusion is that EBSD turns out to be an excellent method to study the stability of $\mathrm{ZrO}_{2}$ dental implant abutments locally and to detect small amounts of the brittle $\mathrm{m}-\mathrm{ZrO}_{2}$ phase. Moreover details of the microstructural characteristics such as grain size distribution, tetragonal and monoclinic phase crystal orientation relationships and technological texture are provided.

As far as the details are concerned we conclude that the average grain size of the tetragonal phase in the pristine ZirDesign samples was larger than the average grain size of the tetragonal phase in the pristine Atlantis implant abutments, being both well below $500 \mathrm{~nm}$. Texture has been detected in both types of Atlantis and ZirDesign abutments. The preferred orientation of tetragonal $\mathrm{ZrO}_{2}$ nanocrystalline grains is induced in the manufacturing steps during abutment fabrication.

An important finding is that none of the samples showed an increasing volume fraction of the monoclinic phase after one year of clinical use. All measured values were far below $25 \%$, the critical value for catastrophic embrittlement. The lowest measured amount of monoclinic phase was 0.5 vol.\% and the highest 8.8 vol.\%. The pristine ZirDesign dental implant abutments showed a higher average volume percentage of the monoclinic phase (6.44 vol.\%) than the Atlantis (1.16 vol.\%) dental abutments. Also, the EBSD scans at different scan areas did not reveal more monoclinic phase at critical places with expected stress concentrations.

It is fair to say that a disadvantage of EBSD is the destructive character of sample preparation damaging both the dental abutment and the attached artificial crown. This precludes a direct comparison of the same sample before and after clinical use. Also the nonconductive nature of Y-TZP caused charging during the EBSD scanning at "standard" 
conditions. This means that one has to make scans of smaller areas and use relatively high frame collection rate. Reducing the SEM acceleration voltage is also important. The $25 \times 25$ $\mu \mathrm{m}^{2}$ sized scans with $50 \mathrm{~nm}$ point spacing were possible without map distortion after reducing the acceleration voltage to $10 \mathrm{kV}$.

\section{ACKNOWLEDGEMENTS}

This study was supported by a grant from the Dutch Society for Gnathology and Prosthetic Dentistry (Nederlandse Vereniging voor Gnathologie and Prothetische Tandheelkunde, NVGPT), Dentsply Sirona Implants, Mölndal, Sweden and by the authors' institutions. Restorative materials were provided by Dentsply Sirona Implants free of charge. All authors approved the final version of the manuscript, agree to be accountable and declare no conflict of interest.

\section{REFERENCES}

[1] Graeve, O.A., Zirconia. Ceramic and Glass Materials, Structure, Properties and Processing. Springer: New York, pp. 169-197, 2008.

[2] Fabris, S., A stabilization mechanism of zirconia based on oxygen vacancies only. Acta Mater, 50(20), pp. 5171-5178, 2002.

[3] Chen, Y.W., Moussi, J., Drury, J.L. \& Wataha, J.C., Zirconia in biomedical applications. Expert Rev. Med. Devices, 13(10), pp. 945-963, 2016.

[4] Van Brakel, R., Noordmansm H J., Frenken, J., de Roode, R., de Wit. G.C. $\&$ Cune, M.S., The effect of zirconia and titanium implant abutments on light reflection of the supporting soft tissues. Clin. Oral Implants Res. 22(10), pp. 1172-1178, 2011.

[5] Denry, I. \& Kelly, J.R., State of the art of zirconia for dental applications. Dent. Mater. 24(3), pp. 299-307, 2008.

[6] Pereira, G.K.R. et al., Low-temperature degradation of Y-TZP ceramics: A systematic review and meta-analysis. J. Mech. Behav. Biomed. Mater. 55, pp. 151-63, 2016.

[7] Chevalier, J., Gremillard, L. \& Deville, S., Low-temperature degradation of Zirconia and implications for biomedical implants. Annu. Rev. Mater. Res, 37 pp. 1-32, 2007.

[8] Li, J.F. \& Watanabe, R., Phase transformation in Y 20 3-partially-stabilized ZrO 2 polycrystals of various grain sizes during low-temperature aging in water. J. Am. Ceram. Soc. 81(10), pp. 2687-2691, 1998.

[9] Schwartz, A.J., Kumar, M., Adams, B.L. \& Field, D.P., Electron Backscatter Diffraction in Materials Science. Springer US: Boston, MA, 2009.

[10] Deville, S., Gremillard, L., Chevalier, J. \& Fantozzi, G., A critical comparison of methods for the determination of the aging sensitivity in biomedical grade yttriastabilized zirconia. J. Biomed. Mater. Res. - Part B Appl. Biomater. 72(2), pp. 239-245, 2005.

[11] Anon Dentsply UK Website.

[12] Li, K.C., et al., Effect of autoclave induced low-temperature degradation on the adhesion energy between yttria-stabilized zirconia veneered with porcelain. Dent. Mater. 29, pp. 263-270, 2013.

[13] Suzuki-Muresan, T., Deniard, P., Gautron, E., Petricek, V., Jobic. S \& Grambow, B., Minimization of absorption contrast for accurate amorphous phase quantification: application to ZrO2 nanoparticles. J. Appl. Crystallogr, 43, pp. 1092-1099, 2010.

[14] Berek, H., Yanina, A., Weigelt, C. \& Aneziris, C.G., Determination of the Phase Distribution in Sintered TRIP-Matrix / Mg-PSZ Composites using EBSD. Steel Res. Int. 82(9), pp. 1094-1100, 2011. 
[15] Kelly, P.M. \& Rose, L.R.F., The martensitic transformation in ceramics - its role in transformation toughening. Prog. Mater. Sci., 47, pp. 463-557, 2002.

[16] Cayron, C., Douillard, T., Sibil, A., Fantozzi, G. \& Sao-Jao, S., Reconstruction of the Cubic and Tetragonal Parent Grains from Electron Backscatter Diffraction Maps of Monoclinic Zirconia. J. Am. Ceram. Soc. 93(9) pp. 2541-2544, 2010.

[17] Mitov, G., Heintze, S.D., Walz, S., Woll, K., Muecklich, F. \& Pospiech, P., Wear behavior of dental Y-TZP ceramic against natural enamel after different finishing procedures. Dent. Mater, 28, pp. 909-918, 2012.

[18] Brisset, F., Using a controlled pressure field emission gun scanning electron microscope to acquire EBSD data on non-conductive samples. IOP Conf. Ser. Mater. Sci. Eng, 32(1), 2012.

[19] Schwarzer, R.A. \& Hjelen, J., Orientation microscopy with fast EBSD. Mater. Sci. Technol. 26(6), pp. 646-649, 2010.

[20] Saraf, L.V., Dependence of the Electron Beam Energy and Types of Surface to Determine EBSD Indexing Reliability in Yttria-Stabilized Zirconia. Microsc. Microanal, 18(2), pp. 371-378, 2012.

[21] Saowadee, N., Agersted, K. \& Bowen, J.R., Effects of focused ion beam milling on electron backscatter diffraction patterns in strontium titanate and stabilized zirconia. J. Microsc. 246(3), pp. 279-286, 2012.

[22] Podlesak, H., Łatka, L., Dietrich, D., Pawłowski, L., Wielage, B. \& Lampke, T., Electron microscopy and diffraction studies of suspension-plasma-sprayed ZrO2+8wt.\% Y2O3 coatings. Surf. Coat. Technol. 220, pp. 67-73, 2013.

[23] Pezzotti, G., Munisso, M.C., Porporati, A.A. \& Lessnau, K., On the role of oxygen vacancies and lattice strain in the tetragonal to monoclinic transformation in alumina/zirconia composites and improved environmental stability. Biomaterials, 31(27). pp. 6901-6908, 2010.

[24] Pee, J.H., Tada, M. \& Hayakawa, M., Crystallographic study of the isothermal and athermal martensites of yttria-doped zirconia. Mater. Sci. Eng, A 438-440, pp. 379-382, 2006.

[25] Keuper, M., Berthold, C. \& Nickel, K.G., Long-time aging in 3mol.\% yttria-stabilized tetragonal zirconia polycrystals at human body temperature. Acta Biomater, 10(2), pp. 951-959, 2014.

[26] Keuper, M., Eder, K., Berthold, C. \& Nickel, K.G., Direct evidence for continuous linear kinetics in the low-temperature degradation of Y-TZP. Acta Biomater, 9, pp. 4826-4835, 2013. 